\title{
Empowerment, satisfacción laboral e identificación organizacional en funcionarios andaluces
}

Josué Rico-Picó, Ángela Peinado-Estévez, Ma Reyes Salvador-Ruiz y Francisco GonzálezFuentes. Universidad de Granada

\author{
Recepción: 20 de mayo de 2016 | Revisión: 21 de mayo de 2016 | Aceptado: 30 de mayo de 2016 \\ Correspondencia: josuerico@correo.ugr.es \\ Citar: Rico-Picó, J., Peinado-Estévez, A., Salvador-Ruiz, MR. y González-Fuentes. F. (2016). Empowerment, \\ satisfacción laboral e identificación organizacional en funcionarios andaluces. ReiDoCrea, 5(2), 33-39.
}

\begin{abstract}
Resumen: En el presente estudio se ha intentado comprobar cómo interactúan el empowerment psicológico, la satisfacción laboral y la identificación laboral. Además, se exploraron las consecuencias de la disminución de la retribución económica en el empowerment. Para ello, seleccionamos una muestra de diferentes grupos laborales que se habían visto afectados de manera desigual tras la crisis, concretamente: docentes, sanitarios y cuerpos de seguridad del estado. A partir de los datos obtenidos comprobamos que el componente económico del apoyo sociopolítico no es suficiente para predecir variaciones en el empowerment, así como tampoco variabilidad en la satisfacción e identificación de cada grupo. No obstante, sí que encontramos una relación significativa positiva entre satisfacciónempowerment e identificación-empowerment, tal y como se esperaba, pero no entre identificación-satisfacción.
\end{abstract}

Palabras clave: Satisfacción | Empoderamiento

Empowerment, Job Satisfaction and Organizational Identification in Andalucian Civil Servants

Abstract: The aim of this study was to determine how psychological empowerment, work satisfaction and organizational identification interact. In addition, we explored how reduction in wages and benefits affected empowerment. To this end, we selected a sample of teachers, public health and law enforcement workers, who were affected in different ways by the financial crisis. The results obtained show that the economic aspect of socio-political support is not sufficient to predict the variability of empowerment, satisfaction Aor identification for each group. However, we did find a significant positive relationship between satisfaction-empowerment and identificationempowerment, as expected, but not between identification-satisfaction.

Key words: Satisfaction | Empowerment

\section{Introducción}

Desde que Kanter en 1977 introdujese el término de empowerment en el ámbito laboral, éste ha sido ampliamente estudiado. Esto se debe a su relación con numerosas actitudes positivas y tiene, por tanto, una amplia implicación en el clima organizacional. Concretamente, se le ha caracterizado como un constructo multinivel en el que se distinguen 3 planos: individual, organizacional y comunitario, que interaccionan entre sí (Mendoza, 2002).

En este estudio nos vamos a centrar en el plano individual del empowerment y únicamente en su componente psicológico. Este concepto hace referencia a cómo las personas dentro de una organización tienen sensación de autonomía, control, competencia e impacto (Spreitzer, 1995). Estas 4 cogniciones se relacionan con las teorías motivacionales y se podrían definir de la siguiente forma:

- Significado: evaluación personal de la relación entre el trabajo y las expectativas o estándares esperados (Hackman y Oldman, 1980).

- Autodeterminación: libertad para desempeñar o regular las tareas en el puesto de trabajo (Deci, Conell y Ryan, 1989).

- Competencia: sentimiento individual de autoeficacia a la hora de desarrollar una tarea requerida (Bandura, 1989).

- Impacto: creencia individual basada en la percepción sobre la capacidad de influir en la administración o grupo de trabajo (Ashforth, 1989). 
Por otro lado, el empowerment psicológico ha sido asociado con diversos antecedentes. Entre ellos, podemos encontrar el apoyo socio-político; las prácticas de dirección; el tipo de liderazgo (Zhu et al., 2012) y las características del trabajo (Meyerson y Kline, 2008). Para nuestro estudio, vamos a centrarnos en el primero, que se refiere a los elementos del contexto provistos por la empresa. De esta forma, cuando a un empleado se le dota de recursos, tanto materiales como sociales o psicológicos, hace que éste perciba un mayor nivel de preocupación por parte de la organización y que aumente la confianza (Taylor, 2007). Es por ello, que dependiendo la retribución económica es posible que afecte a su empowerment psicológico. De otra manera, el empowerment psicológico se ha relacionado con una serie de consecuencias actitudinales, principalmente satisfacción laboral, compromiso organizacional, estrés e intenciones de dimisión (Spreitzer, 1995; Pearson y Moomaw, 2005). En este estudio nos limitamos a la satisfacción laboral, que se define con un alto consenso como un estado emocional positivo dependiente de la percepción entre las características del trabajo y sus expectativas del mismo (Locke, 1976). Ésta relación ha sido demostrada a través de los diferentes estudios y suele tener una correlación positiva significativa (Seibert, 2012). A pesar de no haberse estudiado de forma directa, en ocasiones se ha visto que el empowerment psicológico se puede relacionar con la identificación organizacional como un mediador de influencia (Koberg et al., 1999). Ésta se puede definir como el concepto que una persona tiene de sí misma, atribuyéndose características de la organización a la que pertenece (Ertürk, 2010) y cuenta con dos componentes principales: cognitivos y emocionales. Éstos hacen referencia a compartir intereses, características esenciales y sentimientos positivos, principalmente. Así pues, se ha visto que los antecedentes del empowerment psicológico, como la percepción de respaldo por parte de la organización que tiene el trabajador o la confianza en el supervisor, hacen también que la persona sienta un mayor nivel de identificación grupal. Finalmente, también se ha visto relacionada con algunas de las consecuencias que se le atribuyen al empowerment, por ejemplo, una correlación positiva con la satisfacción y el compromiso organizacional.

En este estudio trataremos de comprobar si los recortes en diferentes áreas laborales (sanidad, docencia y fuerzas y cuerpos de seguridad del estado) generan diferencias significativas. A partir de datos extraídos de la web de la junta de Andalucía se han podido obtener las retribuciones de los funcionarios según el grupo al que pertenezcan. Así, se puede observar que el sueldo base del personal sanitario y docente se ha visto disminuido entre un 2.7 a un $4 \%$, mientras que los cuerpos de seguridad del estado han sufrido una pérdida del $2 \%$, sin contar los suplementos. Por tanto, y de acuerdo con lo expuesto anteriormente, nuestras hipótesis serán las siguientes:

- Hipótesis 1: El empowerment psicológico variará en función de las diferencias salariales de los diferentes grupos.

- Hipótesis 2: La satisfacción laboral y la identificación organizacional tendrán correlación positiva con el empowerment.

- Hipótesis 3: La satisfacción laboral y la identificación organizacional correlacionarán entre sí de forma positiva.

\section{Método}

\section{Participantes}

La muestra total está compuesta por 31 sujetos, 15 hombres y 16 mujeres, pertenecientes a tres colectivos laborales diferentes: personal sanitario (7), docentes 
(10) y fuerzas y cuerpos de seguridad del estado (14). La media de edad total fue de 46 años, con una desviación típica de 7.49, y la antigüedad media en el puesto 20.1 con una desviación típica de 8.92 .

\section{Instrumentos}

Para evaluar el empowerment psicológico empleamos la adaptación española de la Escala de Empowerment Psicológico de Spreitzer (1995). Ésta consta de un total de 12 ítems repartidos en las 4 dimensiones que este autor postula: competencia, significación, impacto y autodeterminación. La puntuación de los ítems se realiza mediante una escala tipo de Likert de 7 puntos, desde totalmente en desacuerdo (1) hasta totalmente de acuerdo (7). La fiabilidad de este instrumento se demuestra con su valor en el alfa de Cronbach de 0.85 (Albar et al., 2012).

La identificación organizacional se evaluó con la Escala de Identificación Grupal de Tarrant, que proporciona datos sobre 3 factores: autocategorización, valoración y compromiso grupal (Cava et al., 2011). Ésta escala tiene una fiabilidad de 0.87 según el alfa de Cronbach como mostró Tarrant (2012). Cuenta con un total de 13 ítems tipo Likert que van de 0 (completamente en desacuerdo) a 10 (completamente de acuerdo).

El cuestionario que se empleó para medir la satisfacción laboral fue el S10/12 elaborado por Meliá y Peiró en 1998. Éste cuenta con, además de una serie de datos demográficos y descriptivos, 12 ítems puntuados en una escala tipo Likert de 7 puntos que oscilan desde 1 (muy insatisfecho) hasta 7 (muy satisfecho). La fiabilidad que arroja es de 0.88 (alfa de Cronbach).

Como método de recogida de datos se empleó la plataforma online Esurvey en la que se introdujeron los ítems e instrucciones correspondientes a cada cuestionario.

\section{Procedimiento}

La metodología empleada es de tipo cuasi-experimental debido a que la asignación de los participantes a cada uno de los grupos se realizó de forma no aleatoria y según los criterios establecidos. Además, estos grupos corresponderían a los distintos niveles de VI utilizados (diferente apoyo económico). Por otro lado, las VD evaluadas fueron la satisfacción laboral, el empowerment psicológico y la identificación grupal.

La técnica de muestreo empleada fue no probabilístico intencional y, posteriormente, bola de nieve. De esta forma los cuestionarios se administraron de forma online a conocidos correspondientes a cada uno de los grupos, quienes a su vez buscaron otros participantes que perteneciesen a su mismo colectivo y dentro de la misma Comunidad Autónoma (Andalucía). El período de recogida de datos está comprendido entre los días 11 y 28 de noviembre de 2015.

Los participantes únicamente tenían que contestar a los ítems de cada uno de los cuestionarios en la plataforma online, pudiendo avanzar únicamente si respondían a todos ellos, de forma completamente individual y voluntaria.

\section{Resultados}

A continuación se muestran los diferentes análisis de los datos tras realizar diversas pruebas en el paquete estadístico SPSS versión 19. La obtención de las puntuaciones totales se realizó siguiendo las indicaciones de los autores de cada escala. Así, para 
todos ellos, este cálculo consistió en una media de las respuestas proporcionadas en la escala tipo Likert. En función del grupo, podemos observar cómo la diferencia numérica entre las poblaciones es mínima, únicamente variando en mayor grado en satisfacción laboral entre el personal sanitario y los docentes y FFCCSE, sin que estas diferencias sean significativas (véase tabla 1 ).

\begin{tabular}{|lcccc|}
\hline Tabla 1. Estadísticos descriptivos & & & \\
\hline \multirow{3}{*}{ Satisfacción laboral } & Sector Laboral & $\mathrm{N}$ & Media (m) & Desviación Típica (dt) \\
\cline { 2 - 5 } & Sanidad & 7 & 3.27 & 1.59 \\
& Docencia & 10 & 4.85 & 0.96 \\
Empowerment & FFCCSE & 14 & 4.32 & 1.70 \\
\cline { 2 - 5 } & Sanidad & 7 & 5.49 & 1.26 \\
Identificación Grupal & Docencia & 10 & 5.81 & 0.83 \\
& FFCCSE & 14 & 5.88 & 0.81 \\
\cline { 2 - 5 } & Sanidad & 7 & 8.33 & 2.13 \\
& Docencia & 10 & 8.19 & 1.57 \\
& FFCCSE & 14 & 8.31 & 2.15 \\
\hline
\end{tabular}

En lo que respecta a las relaciones entre estas variables dependientes, se realizaron coeficientes de correlación de Pearson, indicando éstos que existe relación entre los niveles de empowerment y satisfacción $(r=.477, p=.007)$ y entre el empowerment y la identificación $(r=.644, p=.000)$, sin embargo, no se da una relación significativa entre identificación y satisfacción $(r=.296, p=.106)$. (Véase la tabla 2)

\begin{tabular}{|c|c|c|c|}
\hline \multicolumn{4}{|c|}{ Tabla 2. Correlaciones entre las VD } \\
\hline & Satisfacción Laboral & Identificación Grupal & Empowerment Psicológico \\
\hline Satisfacción laboral & - & .296 & $.477^{\star}$ \\
\hline Identificación grupal & .296 & - & $.644^{* *}$ \\
\hline Empowerment psicológico & $.477^{*}$ & $.644^{* *}$ & - \\
\hline
\end{tabular}

A fin de comprobar la veracidad de las hipótesis planteadas, se calculó si existían diferencias significativas entre las puntuaciones obtenidas por cada grupo en cada una de las variables dependientes (Satisfacción Laboral, Identificación Grupal y Empowerment Psicológico). Este contraste se llevó a cabo por medio de un ANOVA aplicado a cada una de ellas, que resultó no ser significativo en ninguno de los casos. Para la Satisfacción Laboral $(F(2,28)=.013, p=.987)$, la Identificación Grupal $(F(2,28)=2.376, p=.111)$ y el Empowerment $(F(2,28)=.427, p=.657)$. Los resultados de esas puntuaciones se representan en el Gráfico 1.

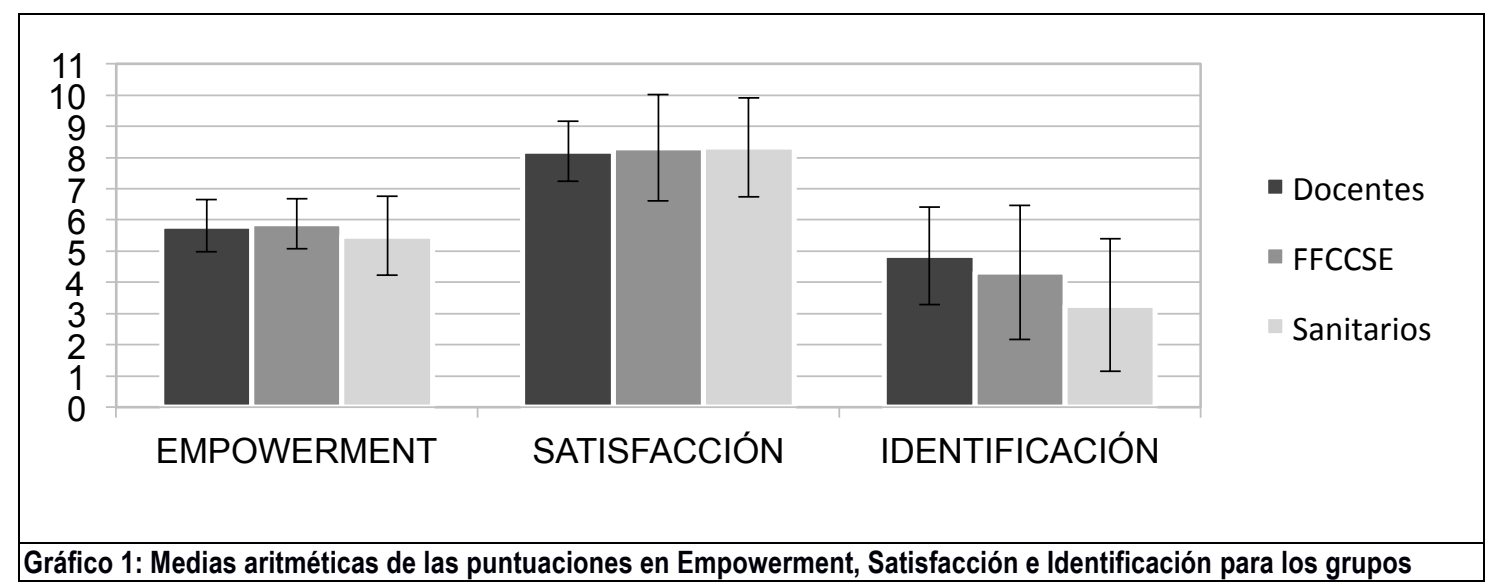




\section{Discusión}

Según la Hipótesis 1, cabía esperar que, a consecuencia de los diferentes recortes aplicados a cada uno de los colectivos, éstos obtuviesen distintas puntuaciones en empowerment, satisfacción e identificación. No obstante, los datos y análisis no indican que haya una variabilidad significativa entre ellos. Esta información indica que la retribución económica parece no tener suficiente importancia para predecir los resultados en empowerment y sus consecuencias. Sin embargo, no debemos olvidar que hay otros factores antecedentes que influyen en éste y, además, que dentro de los socio-políticos hay otros moduladores. Así, dentro de éstos es también relevante el apoyo social y psicológico que le proporcione la organización, que, si se tienen en cuenta en conjunto, sí producen una correlación positiva según los distintos análisis (Seibert, 2011; Meyerson y Kline, 2008). Por tanto, no podemos descartar que la retribución económica no tenga ningún efecto al no haber tenido en cuenta los otros factores dentro de la misma.

En lo referente a las relaciones entre los tres constructos, encontramos que se cumplen aquellas pertenecientes a la hipótesis 2 .

En primer lugar, la correlación que se da entre satisfacción laboral y empowerment es significativa y positiva, como ya se ha mostrado en diversos estudios (Seibert, 2011; Chang et al., 2010). Esto se puede deber, según diversos autores, a que la satisfacción laboral depende de la significancia, necesidad de desarrollo en el puesto y de experiencias de autonomía en el trabajo (Deci y Ryan, 1985; Hackman y Oldham, 1980; Pearson y Moomaw, 2005). Es decir, para que se produzca satisfacción laboral son necesarios varios componentes que también son centrales en el empowerment psicológico. Por tanto, el sentirse empoderado puede actuar como un mediador al estar formado por las cogniciones referentes a la evaluación de las experiencias clave para que se produzca la satisfacción en el ámbito laboral (Wang y Lee, 2012).

En segundo lugar, la identificación organizacional también correlaciona de forma positiva y significativa con el empowerment psicológico. No obstante, debido al enfoque correlacional empleado, no podemos asegurar que se trate de un antecedente o una consecuencia del último. Cabe destacar que esta discusión está presente en la literatura sobre el tema y se han propuesto dos posibles explicaciones. Por un lado, hay quienes sostienen que cuando un empleado se siente empoderado tiende a sentirse orgulloso de la organización, y por ello, se verán identificados con ésta (Koberg et al., 1999). De otra manera, según la teoría de la identidad social de Tajfel y Turner (1986), cuando una persona incorpora en su identidad las características propias de la organización será más probable que dirija sus conductas de una forma proactiva hacia ésta, siendo el empowerment un mediador en esa relación. No obstante, consideramos que la hipótesis de Koberg es más plausible debido a que para que se produzca la identificación laboral es necesario que exista un buen clima organizacional y que los empleados puedan participar en la misma. Por tanto, esto creará las diversas cogniciones características del empowerment, que se manifestarán en un sentimiento de identidad hacia la empresa.

En cuanto a la relación entre satisfacción e identificación planteada en la hipótesis 3 , vemos que su relación no resulta significativa y, aunque sí se encuentre una pequeña correlación positiva, no es tan representativa como en el resto de estudios (Van Knippenberg y Sleebos, 2006). Sin embargo, consideramos que pueden existir variables extrañas que hayan afectado a la misma, de forma que cuando a un grupo se le reducen los recursos, la identidad grupal de sus miembros aumenta. Asimismo, la alta distintividad y los altos requisitos para formar parte de ellos también pueden haber influencia a su identidad de forma positiva y, por tanto, puede que la relación 
con la satisfacción se haya reducido por este aumento de identidad organizacional.

Finalmente, cabe destacar las limitaciones metodológicas que se han afrontado. Entre ellas la selección de una muestra pequeña y poco representativa de los tres grupos debido a las dificultades para conseguirla. Esto ha hecho que se obtengan datos demográficos muy homogéneos que dejan fuera a gran parte de la población compuesta por interinos y contratos parciales, por ejemplo, dificultando la generalización de nuestros resultados. Por tanto, sería necesario para próximos estudios recabar una muestra más representativa y, sobre todo, creemos que sería interesante estudiar de forma longitudinal los efectos de la crisis en éstos para ver los cambios producidos antes y después de ésta.

\section{Referencias}

Albar, M.J., García-Ramírez, M., López-Jiménez, A.M., \& Garrido, R. (2012). Spanish adaptation of the scale of psychological empowerment in the workplace. The Spanish Journal of Psychology, 15(2), 793-800.

Ashforth, B.E. (1989). The experience of powerlessness in organizations. Organizational Behavior and Human Decision Processes, 43, 207-242.

Bandura, A. (1989). Human agency in social cognitive theory. American Psychologist, 44, 1175-1184.

Cava, M.J., Buelga, S., Herrero, J. y Musitu, G. (2011). Estructura factorial de la adaptación española de la Escala de Identificación Grupal de Tarrant. Psicothema, 23(4), 772-777.

Chang, L.C., Shih, C.H., \& Lin, S.M. (2010). The mediating role of psychological empowerment on job satisfaction and organizational commitment for school health nurses: A cross-sectional questionnaire survey. International Journal of Nursing Studies, 47, 427-433.

Deci, E.L., \& Ryan, R.M. (1985). Intrinsic motivation and selfdetermination in human behavior. New York: Springer.

Deci, E. L., Connell, J. P., y Ryan, R. M. (1989). Self-determination in a work organization. Journal of Applied Psychology, 74, 580-590.

Ertürk, A. (2010). Exploring predictors of organizational identification: Moderating role of trust on the associations between empowerment, organizational support, and identification. European Journal of Work and Organizational Psychology, 19(4), 409-441.

Hackman, J.R., \& Oldham, G.R. (1980). Work Redesign. Reading, Boston: Addison-Wesley.

Kanter, R.M. (1997). Men and women of the corporation. New York: Basic Books.

Koberg, C., Boss, W., \& Goodman, E. (1999). Antecedents and outcomes of empowerment. Group and Organizational Management, 24, 71-91.

Locke, E. A. (1969). What is Job Satisfaction? Behavior and Human Performance, 4, 309-336.

Meliá, J.L. y Peiró, J.M. (1988). El cuestionario de satisfacción S10/12: estructura factorial, fiabilidad y validez. Madrid: Colegio Oficial de Psicólogos de Madrid.

Mendoza, M.I. (2002). El empowerment psicológico en el trabajo. Trabajo: Revista de la Asociación Estatal de Centros Universitarios de Relaciones Laborales y Ciencias del Trabajo, 11, 157-168.

Meyerson, S.L., \& Kline, T.J. (2008). Psychological and environmental empowerment: Antecedents and consequences. Leadership \& Organization Development Journal, 29(5), 444-460.

Pearson, L.C., \& Moomaw, W. (2005). The relationship between teacher autonomy and stress, work satisfaction, empowerment, and professionalism. Educational Research Quartely, 29(1), 38-54.

Seibert, S.E., Wang, G., \& Courtright, S.H. (2011). Antecedents and consequences or psychological and team empowerment in organizations: A meta-analytic review. Journal of Applied Psychology, 96(5), 981-1003. 
Tajfel, H., \& Turner, J.C (1986). The social identity theory of intergroup behaviour. Chicago: Nelson.

Taylor, S.E. (2007). Foundations of health psychology. New York: Oxford University Press.

Van Knippenberg, D., \& Sleebos, E. (2006). Organizational identification versus organizational commitment: Selfdefinition, social exchange, and job attitudes. Journal of Organizational Behavior, 27, 571-584.

Wang, G., \& Lee, P. D. (2009). Psychological empowerment and job satisfaction: An analysis of interactive effects. Group \& Organization Management, 34(3), 271-296.

Zhu, W., Sosik, J.J., Riggio, R.E., \& Yang, B. (2012). Relationships between transformational and active transactional leadership and followers' organizational identification: The role of psychological empowerment. Journal of Behavioral and Applied Management, 13(3), 186-212. 